\title{
El atado de remedios de un religioso/médico del periodo Tiwanaku: miradas cruzadas y conexiones actuales
}

Le sac-pharmacie d'un religieux/guérisseur de la période Tiwanaku: regards croisées et connexions actuelles

The sac-pharmacie belonging to a shaman/priest of the Tiwanaku culture: archaeological and ethnographic insights

\section{Carmen Beatriz Loza}

\section{OpenEdition \\ Journals}

Edición electrónica

URL: http://journals.openedition.org/bifea/3563

DOI: 10.4000/bifea.3563

ISSN: 2076-5827

Editor

Institut Français d'Études Andines

Edición impresa

Fecha de publicación: 1 diciembre 2007

Paginación: 317-342

ISSN: 0303-7495

Referencia electrónica

Carmen Beatriz Loza, «El atado de remedios de un religioso/médico del periodo Tiwanaku: miradas cruzadas y conexiones actuales », Bulletin de l'Institut français d'études andines [En línea], 36 (3) | 2007, Publicado el 01 junio 2008, consultado el 01 diciembre 2020. URL : http://journals.openedition.org/ bifea/3563 ; DOl : https://doi.org/10.4000/bifea.3563

\section{cc)}

Les contenus du Bulletin de l'Institut français d'études andines sont mis à disposition selon les termes de la licence Creative Commons Attribution - Pas d'Utilisation Commerciale - Pas de Modification 4.0 International. 


\title{
El atado de remedios de un religioso/médico del periodo Tiwanaku: miradas cruzadas y conexiones actuales
}

\author{
Carmen Beatriz Loza*
}

\section{Resumen}

Este artículo realiza un análisis detallado de un atado fabricado en cuero de ciervo, denominado watasqa en quechua, conteniendo un gran numero de remedios. Éstos están envueltos, liados y reagrupados en otros cueros. El atado perteneciente a un religioso/médico del periodo Tiwanaku fue descubierto en el sitio Pallqa, provincia de Larecaja del Departamento de La Paz. El watasqa de Pallqa, actualmente conservada por la Unidad Nacional de Arqueología de Bolivia (UNAR), es excepcional para cualquier investigador interesado en las prácticas terapéuticas de la cultura Tiwanaku.

En este trabajo inédito, nos hemos propuesto mostrar la importancia ritual y curativa de ese material, aspecto que no había sido abordado anteriormente. Partiendo de una aproximación que privilegia la analogía etnográfica, presentamos una nueva visión del contenido del atado apoyándonos en los conocimientos de dos categorías de expertos indígenas: los religiosos/médicos y herbolarios kallawaya de la provincia Bautista Saavedra de La Paz y las boticarias/ritualistas k'awayu de la provincia Tomás Frías de Potosí. En el interior del atado se ha podido identificar una jerarquía de cueros empleados para conservar los objetos destinados a la inhalación de polvos alucinógenos y una tableta decorada de gran tamaño con estuche completo. El aporte sustancial ha consistido en identificar los remedios y los preparados de un gran valor simbólico en el proceso para curar y aliviar diferentes enfermedades/ padecimientos/infortunios y la utilización de textiles. Encontramos artefactos personales del religioso/ médico aproximadamente entre 700 y +1500 después de JC. Son descritos los textiles y analizados los cordajes en sus respectivos contextos, mostrando además los preparados conservados en una organización jerarquizada de recipientes en cuero, cuyos posibles empleos en el proceso curativo son analizados sobre la base de datos recogidos en el trabajo de campo.

Palabras clave: instrumentos médicos, ayuda mnemónico-terapéutica, parafernalia ritual, textiles, simbolismo, Tiwanaku, Kallawaya, La Paz, Potosí 


\title{
Le sac-pharmacie d'un religieux/guérisseur de la période Tiwanaku : regards croisées et connexions actuelles
}

\author{
Résumé
}

Cet article fait une analyse détaillée d'un sac en cuir de serf, nommé en quechua watasqa, qui contient de nombreux remèdes enveloppés dans des petits étuis. Le sac, qui appartenait à un religieux/guérisseur de l'époque Tiwanaku, fut découvert sur le site de Pallqa, province de Larecaja, département de La Paz. Cet objet, actuellement conservé par I'Unidad Nacional de Arqueología de Bolivia (UNAR) est exceptionnel pour quiconque s'intéresse aux pratiques thérapeutiques de la culture Tiwanaku.

Dans ce travail inédit, nous nous proposons de montrer l'importance rituelle et curative de ce matériel, ce qui n'avait pas été fait jusqu'à présent. À partir d'une approche qui privilégie l'analogie ethnographique, nous présentons une nouvelle façon de voir le contenu du sac, en nous appuyant sur les savoir-faire de deux catégories d'experts indigènes : les religieux/médecins et herboristes Kallawaya de la province Bautista Saavedra de La Paz, et les femmes apothicaires aymara K'awayu de la province Tomás Frías de Potosí, spécialistes et distributeurs d'objets symboliques et de plantes médicinales. À l'intérieur du sacpharmacie on a pu identifier une hiérarchie de récipients en cuir utilisés pour conserver des substances destinées à l'inhalation de poudres hallucinogènes lors des rituels, ainsi qu'une tablette à priser décorée de grande taille avec un étui complet. Nous avons également identifié des remèdes et des préparations d'une grande valeur symbolique pour guérir et soulager différentes maladies, souffrances et malheurs, ainsi que des textiles utilisés par un seul homme, approximativement entre 700 et 1500 après J.C. Sont décrits les textiles, les cordelettes et les préparations conservées dans un emboîtement hiérarchisé d'étuis en cuir dont les emplois possibles dans le processus curatif sont analysés sur la base des données recueillies lors du travail de terrain.

Mots clés : instruments médicaux, aide mnémonique-thérapeuthique, ensemble d'objets rituels, textiles, symbolisme, Tiwanaku, Kallawaya, La Paz, Potosí

\section{The sac-pharmacie belonging to a shaman/priest of the Tiwanaku culture: archaeological and ethnographic insights}

\begin{abstract}
In this article, we analyze an exceptional ritual find from the Tiwanaku culture. Our intent is to understand the ritual and medicinal relevance of this assemblage, a q'ipichata belonging to a Tiwanaku shaman-priest found at the site of Pallqa in the valley of Amaguaya, Province of Larecaja in the Department of La Paz. We based our analysis on the use of ethnographic analogy, in particular on the understanding of two indigenous specialists: the renowned ritual shamans known as the Kallawaya from the province of Bautista Saavedra of La Paz, and the K'awayu from the province of Tomas Frías of Potosí, specialists and distributors of symbolic objects and medicinal plants. We have been able to identify a hierarchy of hide pouches used to hold and conserve the ritual paraphernalia and the snuff. Notable items include a large decorated snuff tablet, the personal clothing of the individual dated between AD 700-1500, and various medicines with symbolic and medicinal properties.

We begin by analyzing the technological aspects of the artifacts through such qualities as the elements as the hides, the cords and the textiles; afterwards, we looked at the iconographic aspects with the intent of dating and assigning cultural affinity to the artifacts. Finally, we analyze the medical and ritual aspect by identifying each of the medicinal remains. In summary, our analysis takes into consideration the entire assemblage of the q'ipichata and proposes possible uses of the ritual and medicinal remains.
\end{abstract}

Key words: medical instruments, therapeutic memory aids, ritual paraphernalia, textiles, symbolism, Tiwanaku, Kallawaya, La Paz, Potosí 
Son excepcionales, en territorio boliviano, las muestras de cultura material que nos iluminan acerca de los instrumentos, las tecnologías mínimas y las prácticas médicas utilizadas durante el periodo Tiwanaku (Posnansky, 1945; Wassén, 1972). Los estudios se ven dificultados, en parte, por la insuficiente información acerca de los envoltorios, paquetes y atados rituales y su función en la sociedad de ese tiempo. En 1998 fue hallado un envoltorio que creemos perteneció a un religioso/médico ${ }^{1}$ que se desempeñó en el valle de Amaguaya - actual cantón San Juan de Challana en la segunda sección del municipio de Guanay de la provincia Larecaja del departamento de La Paz (Instituto Nacional de Estadística \& PNUD, 2005: 131)—, tal como nos lo plantea la naturaleza de elementos guardados en el atado, aspecto sobre el cual desarrollaremos nuestros principales argumentos a lo largo de este texto.

El estupendo envoltorio de Pallqa —actualmente en custodia en la Unidad Nacional de Arqueología de Bolivia (en adelante UNAR)—, llamó la atención de los arqueólogos por tratarse de un hallazgo superficial que no pertenece a un contexto cultural, enterrado o funerario como mucha de la parafernalia ritual ubicada, principalmente, en San Pedro de Atacama, en el actual norte de Chile (cf. Torres, 1986; 1996; Llagostera, 1988; Llagostera, 2006), sino por estar manifiesto y presentar un buen estado de conservación. De esa manera, los arqueólogos bolivianos efectuaron estudios observando dos aspectos. Por un lado, identificaron los materiales de fabricación, particularmente los pelos y fibras de algunos elementos elaborados con recursos animales (Capriles \& Flores, 2000; 2002; Capriles, 2002). Por otro lado, describieron el ajuar de elementos inhalantes (Rendón, 2000). Es preciso destacar, en ambos estudios, la especialización sobre ciertos aspectos y, en consecuencia, la falta de una panorámica amplia del contenido, su complejidad y su relevancia arqueológica.

Hasta el momento son limitadas las reflexiones sobre los atados y envoltorios en el campo arqueológico boliviano. Numerosos aportes provienen de la literatura etnográfica donde hallamos el inventario de una amplia variedad de formas y funciones en momentos particulares en la vida de los pobladores andinos. Los atados se manifiestan, hasta el presente, en las ceremonias ganaderas de propiciación y fertilidad (Flores Ochoa, 1977; Zorn, 1987: 489-525). Se los ubica preservados en lugares preferenciales de las viviendas o en los propios corrales (Loza-Balsa, 1995: 85). Asimismo, están ubicados en los hitos territoriales entre los grupos más extensos y diferenciados étnicamente (Flores Ochoa, 1977). En ocasiones, esos atados guardan valiosos tejidos y papeles antiguos que han concitado más de una polémica y lucha jurídica por mantenerlos en el seno de sus sociedades (Bubba, 1997; Rivera Cusicanqui, 1986).

En este texto partimos de la premisa de que se trata de un atado de medicinas porque contiene preparados utilizados por el religioso/médico para curar y aliviar diferentes enfermedades/padecimientos/infortunios que aquejaban a los tiwanakutas. Un estudio de este tipo de evidencia debe encarar el difícil tema de su identificación, por esta razón son varias las preguntas planteadas en el análisis: ¿Cómo caracterizar el atado en términos andinos sin necesariamente imponer nuestras propias categorías?, ¿Qué funciones cumplen los artefactos y cuáles son las relaciones existentes entre ellos, según los expertos indígenas

1 A lo largo del texto, empleamos la designación religioso/médico porque no hemos encontrado otra forma para connotar esta doble dimensión, pues creemos que ambas intervienen durante el proceso curativo. Aclaro que no empleo el término de chamán porque se ha convertido en un etiqueta para designar a cualquier doctor indígena (Hultrantz, 2003: 386), pero también porque todavía sigue la controversia «respecto a la forma exacta de clasificar a los chamanes» como «técnicos de lo sagrado» (Lindholm, 2000: 108). A más de esto, entendemos una curación no como de un estado de salud — como cuando se dice «estoy curado»— sino, siempre se hace referencia al proceso, es decir al ritual curativo (Rösing, 1990; 1992; 1995). 
en medicina y ritual que todavía los reconocen o emplean?, ¿Cuáles elementos del atado se mantienen aún en uso y cuáles fueron reemplazados, si es que tal prolongación es concebible sobre una duración de varios siglos?, ¿Qué nivel de análisis es necesario para mostrar una continuidad en su empleo? De todas estas preguntas se tratará más adelante; pero antes hemos de anteponer algunas observaciones.

La complejidad de la evidencia arqueológica y la ausencia de explicaciones globales nos encaminaron a proponer una doble aproximación metodológica de etno-arqueología en acción (David \& Kramer, 2001). Y, en esa vertiente, la analogía etnográfica, apunta a aproximar al lector a la posible función y significación del atado de Pallqa. Somos conscientes de las limitaciones de esta aproximación dentro del enfoque del reconstruccionismo arqueológico, sin embargo, a falta de una mejor alternativa metodológica la empleamos a lo largo del texto. Aclaramos que la reflexión se inscribe en una operación que «cruza» diferentes puntos de vista acerca del atado dentro de un proceso intelectual relacionado con diversos tipos de expertos que expresan su punto de vista en torno a la evidencia arqueológica (cf. Werner \& Zimmermann, 2004). Tal elección se debe al hecho de considerar esencial la «posición del observador» porque supone un punto de vista exterior al objeto; por esa razón, situamos la identificación del atado dentro de filiaciones de categorías culturales andinas y perspectivas distintas a las publicadas.

En esta ocasión se ha partido de la opinión de expertos indígenas itinerantes. Por un lado, tomando en cuenta la mirada de los religiosos/médicos y herbolarios kallawaya de la provincia Bautista Saavedra del departamento de La Paz (Loza, 2004); por el otro, de las boticarias/ritualistas aymaras especializadas en la fabricación y dispensa de medicinas conocidas como qapachaquiras k'awayu del municipio de Urmiri, de la provincia Tomás Frías del departamento de Potosí. Ambos grupos son actualmente trilingües: quechua, aymara y castellano, pero básicamente se valen del quechua, lo que explica la profusión de referencias en este idioma a lo largo del texto. Aunque debemos aclarar que los kallawaya utilizan durante ciertas ceremonias y curaciones su antigua lengua machaj juyai, llamada por algunos autores «lengua secreta de los incas» y por otros simplemente puquina (cf. Oblitas Poblete, 1955; 1956; 1978; Stark, 1972: 199-227; Torero, 1987; Girault, 1984; 1987; Aguiló, 1991).

En ese andar, me relacioné con adultos de ambos sexos kallawaya y k'awayu gracias a una red de contactos sociales variados, porque ellos se complementan, desde hace siglos, en el proceso de referencia y contrarreferencia entre expertos. Al mismo tiempo, a medida que establecía una mejor calidad de relaciones con los entrevistados me autorizaban entrevistas semidirigidas a repetición. En esas ocasiones, el objeto de mis interrogantes se diversificaba: por un lado, la identificación del contenido del atado y por otro lado, al significado de los elementos utilizados actualmente. Las informaciones de los 21 entrevistados sirvieron para incorporar en este texto los datos recogidos principalmente en los departamentos de La Paz y Potosí. Asimismo, las entrevistas, cada vez que fue posible, fueron confrontadas con observaciones «en situación» durante diversas consultas con el objetivo de comparar lo que era dicho al momento de manipular los elementos del atado y lo que mire en las manos expertas de nuestros entrevistados (cf. referencias citadas).

La reconstrucción se basa en la comparación entendida como una operación cognitiva que básicamente funciona según el principio de oposición binario entre diferencia y similitud. A partir de ello, enlazamos al atado con la función y el sentido actual de su empleo. Así, esa conexión tendría sobre todo la virtud de conjeturar una continuidad en el manejo de remedios entendidos como el medio que se toma para reparar un daño o inconveniente para producir un cambio en la salud del enfermo. El hecho de establecer perseverancias nos permitirá, simultáneamente, evidenciar la sustitución de elementos privativos que intervienen en la antigua tradición curativa y ritual. 
Bajo esta perspectiva, a lo largo del texto, reflexionaremos a partir de la dicotomía: continuidad/sustitución en el empleo de los componentes existentes en el envoltorio de Pallqa. En la primera parte presentamos, de manera general, las circunstancias de su localización, enfatizando su caracterización en términos andinos para describir la evidencia arqueológica y comprender el modelo cognitivo que prima en el orden del atado. El problema de la terminología estará presente en los distintos acápites del texto para aclarar aspectos puntuales en el manejo de este tipo de objetos técnicos. En la segunda parte, se presenta una propuesta de comprensión de la organización y almacenamiento y ordenación de los remedios, proponiendo la existencia de una distribución jerárquica de su contenido. En la tercera parte, se presenta el contexto del ajuar inhalatorio. Si bien esta exposición es breve en virtud de que ya ha sido estudiado anteriormente por otros autores (Rendón, 2000: 89-96), enfatizaremos en algunos aspectos descriptivos no tratados precedentemente. Finalmente se revisarán algunas de las principales cuestiones planteadas a lo largo del texto, tomando en cuenta las conclusiones que se pueden sacar de los datos arqueológicos y etnográficos que sustentan nuestros argumentos.

\section{EL ATADO DE REMEDIOS, UNA VISIÓN DE CONJUNTO}

\section{1. Del hallazgo del atado de Pallqa a su custodia en la UNAR}

Entre enero y febrero de 1998, en la comunidad de Amaguaya —el valle del mismo nombre se encuentra a 180 kilómetros de la sede de gobierno- Francisco Pillco Flores, un comunario y autoridad comunal, luego de asistir al oficio religioso evangélico se dirige a la serranía en busca de leña. Desde este valle, Francisco Pilco inicia su caminata y minuciosa inspección por el sector de Chojñakota, trepando hasta la parte media de un afloramiento rocoso donde identifica un abrigo natural pedregoso denominado Pallqa, situado a $4000 \mathrm{~m}$. s.n.m. Inesperadamente, halla una piedra y debajo de ella un antiguo atado de cuero de taruca (AUNAR, 1998a).

Suponemos que el atado fue depositado en el abrigo de Pallqa con la certidumbre de que no corría riesgo alguno, pues exhibía las marcas de un capital simbólico acumulado por el religioso/médico a quien pertenecía; y que, por lo tanto, nadie se atrevería a violentar aquel bulto colocado en ese lugar. Por esa razón, suponemos permaneció cobijado y completamente cerrado desde una época estimada entre 700 y 1500 d.C. hasta 1998 — desconocemos la fecha precisa en que fue guardado—, cuando Pillco Flores, comunario evangélico, se atrevió a abrirlo porque los códigos del atado le eran indiferentes debido a su nueva ideología religiosa.

Pillco Flores desató y desenvolvió el bulto y lo transportó hasta su vivienda donde procedió a substraer las incrustaciones de metal de la tableta con la esperanza de proveerse de unos cuantos gramos de oro; sin embargo pudo percatarse posteriormente de que su presunción había sido errónea, pues solo se trataba de bronce (AUNAR, 1998a). Esto no fue un óbice para proyectar la venta del conjunto de su descubrimiento en La Paz, ciudad capital administrativa y centro económico del país, a la cual se trasladó en compañía de Juan Fernando Mamani, otra autoridad originaria del cantón Challana.

Una vez en La Paz, deambuló infructuosamente ofreciéndolo a la venta. Finalmente, llegó a la UNAR donde Pillco Flores y Mamani fueron convencidos, ante testigos, de dejar en custodia ese material arqueológico para su resguardo y posterior estudio (AUNAR, 1998a; 
1998b)2. Cinco meses después, en junio de 1998, se planificó un proyecto de prospección arqueológica en la zona a través de un convenio de investigación entre la UNAR y el The Cleveland Museum of Art para contextualizar el hallazgo desde el punto de vista arqueológico y medioambiental, llegándose a concretar posteriormente un reconocimiento y excavación en la ladera este del cerro Pallqa, y en un valle ubicado al pie de la mencionada montaña (AUNAR, 1998c; 1998d; 1998e; El Diario, 1998a; 1998b; Presencia 1998a; 1998b). En esa oportunidad, se identificaron, por lo menos, seis abrigos rocosos más — desde aquel primero hallado por Pillco Flores-; la mayoría con rastros de cultura material debido a las muestras de huesos, cueros, semillas, hilos entre otros que se logró recuperar (AUNAR, 1998f; Conde, 1998).

El 22 de septiembre de 1999, el Viceministerio de Cultura de Bolivia y la Fondazione Metropolitan suscribieron un contrato para la exhibición de 237 piezas correspondientes a la cultura Tiwanaku. Formaba parte de ese conjunto el atado de Pallqa (AUNAR, 1998a). Aunque las piezas fueron devueltas a la UNAR en julio de 2001, únicamente en noviembre de 2003 se las expuso, por primera vez en Bolivia, a raíz de que la ciencia y cosmovisión Kallawaya fue declarada como Obra Maestra del Patrimonio Oral e Intangible de la Humanidad por la UNESCO (cf. Loza, 2004). La política de exaltar exhibiciones internacionales sin el aditamento de descripciones y estudios detallados que sirvan de base para las futuras investigaciones, permite explicar el perjuicio en el estudio y retardo en la publicación de aquellas realizadas y su aparición no solo esporádica sino fragmentaria.

\section{2. Los remedios organizados sistemática y jerárquicamente}

El primer problema que nos plantea el hallazgo arqueológico de Pallqa es su definición debido a la ambigüedad y poca inteligibilidad de las diferentes caracterizaciones empleadas con anterioridad: parafernalia, equipo, paquete, bienes y conjunto (Capriles \& Flores, 2002: 133-146; Capriles, 2002: 33-50). Todas ellas no expresan con precisión su identidad y la dimensión operativa y funcional. Se tratará entonces de saber cómo puede ser explicado en términos andinos y de afinar categorías conceptuales para comprender las significaciones de la manipulación de objetos que, desde nuestro punto de vista, están estrechamente vinculadas con la dimensión curativa y religiosa durante el periodo Tiwanaku en los valles del norte paceño.

Defendemos, entonces, el argumento, de que materialmente se trata de un atado de carácter médico/ritual en vista de las características estructurales de su forma material y en virtud de la preponderancia de remedios muy variados. Y fue su propietario quien debió depositar esos objetos perennes que dan fe de su práctica y poder. Por eso mismo los embaló, sujetó con lazos para guardarlos mejor y, quién sabe, para recuperarlos en algún momento a fin de depositarlos en un contenedor especial y transportarlo a su destino. Dadas esas características estructurales nos adherimos más bien a la definición de los kallawaya para quiénes sería más bien un watasqa, es decir un atado liado con funciones de curación simbólica porque está fabricado con piel de taruca, un animal sagrado que reverencian en la cosmovisión andina (para mayores detalles véase el acápite 3 en el texto). En adelante entonces referiremos como el watasqa de Pallqa (ver cuadro 1).

2 La reconstrucción de los hechos se basó en documentación interna e inédita del Archivo de la Unidad Nacional de Arqueología (AUNAR, 1998a; 1998b). Al mismo tiempo, fue complementada con las entrevistas 1 y 2 . En este punto, parece pertinente subrayar que muchos aspectos de los eventos narrados en este texto difieren considerablemente de lo anteriormente publicado sobre el atado (cf. Capriles \& Flores, 2002: 133-146; 2000; Capriles, 2002: 33-37). 
El hallazgo consistió en un envoltorio liado compuesto de un cuero de taruca amorfo, de aproximadamente $38 \mathrm{~cm}$ de largo máximo, que servía de embalaje y protección exterior de una variedad de embalajes intermedios conteniendo una diversidad de componentes. Al desenvolverlo, se trata de un sistema de almacenamiento que rige la organización de contenedores y protectores interiores. Está estructurado a partir de un modelo de pensamiento denominado simbolismo que puede ser descrito como un encajonamiento repetitivo, secuencial y relacional, cuyo principio lógico se asemeja al de las «muñecas rusas», es decir que cada contenedor — sin importar la naturaleza del material— alberga dentro de sí otro elemento que a su vez acoge a otro, ordenándolos en serie. Este sistema se ilustra a cabalidad cuando se trata de cueros. En este entendido, el primer paso es la presentación de los contenedores y «elementos interiores» que van apareciendo de acuerdo a una sucesión encadenada. Comprender los mecanismos implicados en esta tarea necesita de un análisis de su función dentro del marco del dominio cognitivo, donde prevalece el modelo de «muñecas rusas» (cf. Chapelle, 1998). Se justifica además tal presentación porque los análisis anteriores publicados no brindan una descripción secuencial lo suficientemente nítida y prolija para entender la ubicación de los elementos en la medida que no se detuvieron en el protocolo de apertura, el cual debió ser complementado con la información oral de los testigos presenciales en ese proceso (AUNAR, 1998b).

Esta es la base para haber identificado que el modelo de simbolismo presente en el watasqa de Pallqa fue estructurado a partir de un sistema modular, es decir que en cada nivel de ensamblaje de los cueros, se verifica el almacenamiento de los unos en los otros según una organización lógica y jerárquica precisa. De manera que el criterio de tamaño es la base para distinguir cuatro tipos de contenedores: los mayores, intermedios, pequeños y diminutos, conforme se explica a continuación:

\section{2. 1. Los embalajes y contenedores mayores}

Se trata de los contenedores que denominamos cueros-envolventes mayores para connotar una doble significación: sirven desde el punto de vista técnico como embalaje porque acogen la mayor parte de los objetos; mientras que desde el punto de vista médico/ritual su materialidad interviene en el proceso curativo. Hemos podido distinguir los cuerosenvolventes mayores de piel de taruca que sirven de protección exterior. Están asegurados y amarrados con un lazo de sujeción no torcelado fabricado de la fibra de corteza de orochi, que es una planta privativa de los valles mesotérmicos. Los guaraníes chaqueños la utilizan para confeccionar sogas a través del torcelado de la fibra que puede ser de dos o tres cabos para que sea más resistente (APCBL, entrevista 20). En realidad, se trata de un cuero del cuerpo de la taruca al que se añaden dos cueros más de taruca, amorfos, notoriamente diferenciados cada una de ellos por sus dimensiones [CAAGY-01; CAAGY-02], que sirvieron para armar la estructura del atado adaptando a las características del cuero del animal y efectuando un buen embalaje, cuidando escrupulosamente en liar delicadamente todo su contenido, lo que explica que la piel recubriese el interior. Aunque hemos identificado ese material, carecemos de indicios sobre la forma original de la atadura porque no intervino un arqueólogo en el momento de desatarlo y desenvolverlo.

\section{2. 2. Los embalajes y contenedores intermedios}

Son de varios tipos. Por ejemplo, la bolsa policroma, tipo saquillo rectangular, con base semi-deteriorada [CAAGY-05] guardaba gran parte de los objetos y estaba asegurada con un lazo que impedía su salida de los mismos. Se debe mencionar que existía un lazo mixto 
Carmen Beatriz Loza

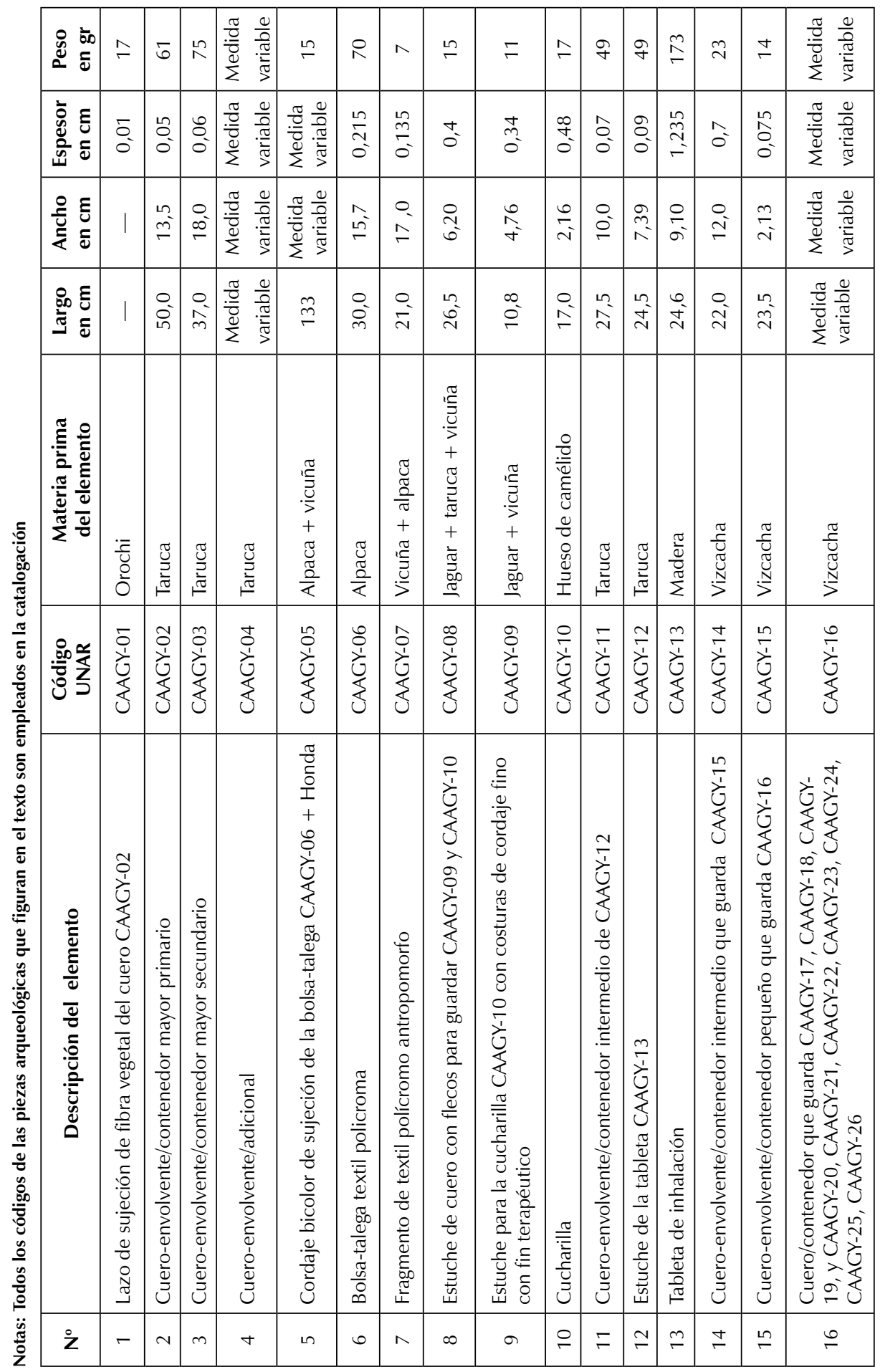


El atado de remedios de un religioso/médico del periodo Tiwanaku

\begin{tabular}{|c|c|c|c|c|c|c|c|c|c|c|c|c|c|c|c|c|c|c|c|c|c|c|}
\hline$\infty$ & 5 & $\overline{0}$ & $\stackrel{m}{-}$ & $\ddot{0}_{0}^{0}$ & $\overline{0}$ & $\tilde{c}$ & $\begin{array}{l}6 \\
0\end{array}$ & $\bar{o}$ & - & $m$ & $\bar{c}$ & $5 \infty$ & 0 & 5 & $n=2$ & & & $\Rightarrow F$ & {$\left[\begin{array}{l}N \\
0\end{array}\right.$} & $\sim$ & $\tilde{N}$ & 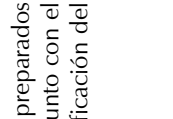 \\
\hline$\stackrel{\text { Oै }}{-}$ & 1 & | & | & $\begin{array}{l}0 \\
0 \\
0\end{array}$ & | & | & $\begin{array}{l}0 \\
0 \\
0\end{array}$ & | & 1 & | & 1 & 1 & 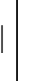 & & c & & & & 1 & $\begin{array}{l}\hat{\imath} \\
-\end{array}$ & & 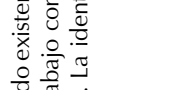 \\
\hline 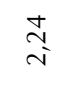 & 1 & | & | & $\begin{array}{l}L 0 \\
\infty \\
1 n\end{array}$ & | & | & 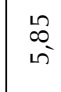 & I & & $\begin{array}{l}8 \\
0 \\
17\end{array}$ & 1 & 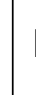 & & 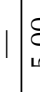 & rit & & & & 1 & $\begin{array}{l}1 \\
\hat{y} \\
v\end{array}$ & & 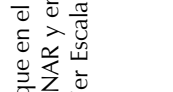 \\
\hline$\frac{\nabla}{\infty}$ & 1 & | & | & $\stackrel{f}{f}$ & $\stackrel{ \pm}{\sim}$ & 1 & $\stackrel{f}{+}$ & $\stackrel{ \pm}{\sim}$ & & เి & 1 & & $\delta$ & 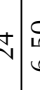 & 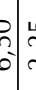 & & & & $\stackrel{ \pm}{\sim}$ & $\begin{array}{l}m \\
\tilde{n} \\
\sim\end{array}$ & $\stackrel{ \pm}{\sim}$ & 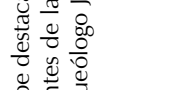 \\
\hline 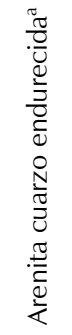 & ט气 & 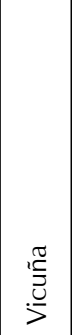 & 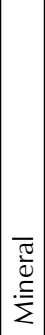 & $\frac{\sqrt{0}}{\frac{\pi}{2}}$ & $\frac{\pi}{\mathbb{Z}} \frac{\pi}{<}$ & $\begin{array}{l}\frac{\pi}{4} \\
\frac{\square}{2} \\
\end{array}$ & 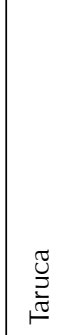 & $\begin{array}{l}\underset{\pi}{0} \\
\frac{\Im}{2}\end{array}$ & $\frac{\pi}{\frac{\pi}{2}}$ & $\left\{\begin{array}{l}\frac{\pi}{\tilde{J}} \\
\frac{\tilde{J}}{N} \\
>\end{array}\right.$ & 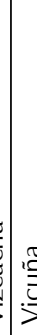 & كَّ & 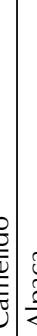 & 赵| & 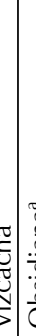 & 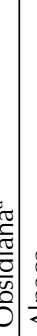 & & 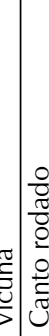 & \begin{tabular}{|l}
0 \\
$\frac{\pi}{\pi}$ \\
$\frac{2}{<}$
\end{tabular} & 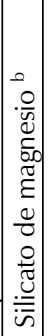 & $\begin{array}{l} \\
\frac{\tilde{\pi}}{\pi} \\
\frac{\pi}{<}\end{array}$ & 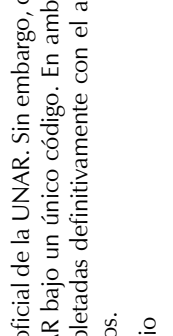 \\
\hline$\frac{n}{\frac{1}{3}}$ & $\frac{\infty}{\frac{\pi}{1}}$ & $\frac{0}{\frac{1}{1}}$ & 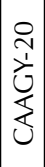 & $\frac{i}{i}$ & $\frac{\pi}{N}$ & $\frac{\tilde{z}}{3}$ & $\frac{d}{\frac{1}{3}}$ & 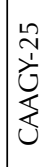 & \begin{tabular}{l}
2 \\
$\frac{1}{3}$ \\
\multirow{3}{3}{}
\end{tabular} & $\frac{1}{3}$ & $\left\{\begin{array}{l}\infty \\
\frac{1}{3} \\
\frac{1}{3}\end{array}\right.$ & 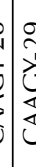 & 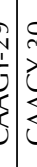 & 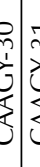 & 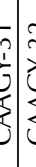 & 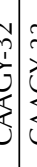 & $\frac{0}{3}$ & 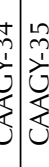 & 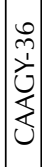 & 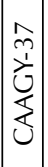 & $\begin{array}{l}\infty \\
m \\
\frac{1}{1} \\
\frac{1}{d} \\
\end{array}$ & 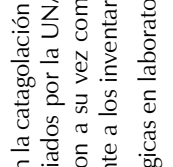 \\
\hline 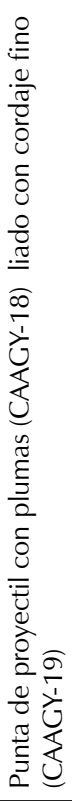 & 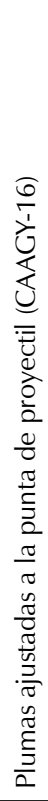 & 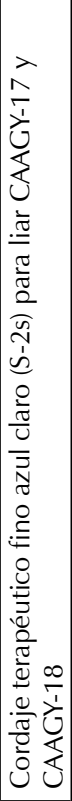 & 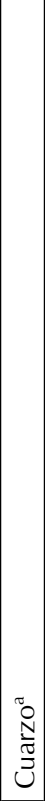 & 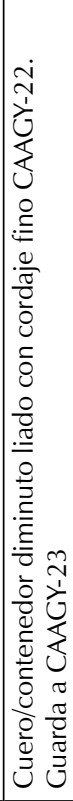 & 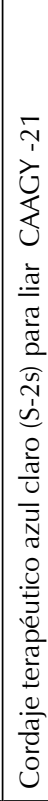 & $\begin{array}{l}0 \\
0 \\
0 \\
0 \\
0\end{array}$ & 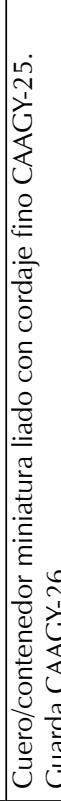 & 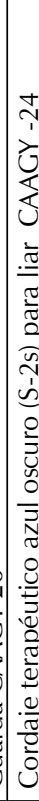 & 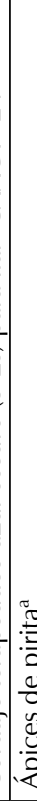 & 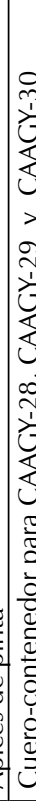 & 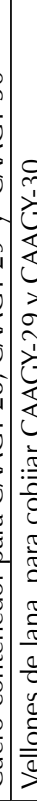 & 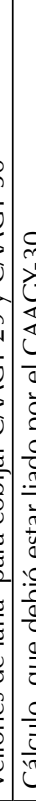 & 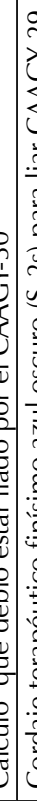 & 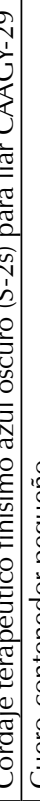 & 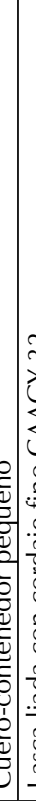 & 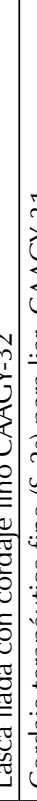 & 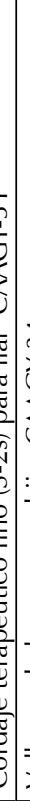 & 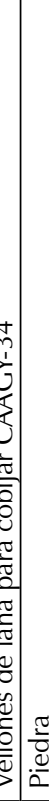 & 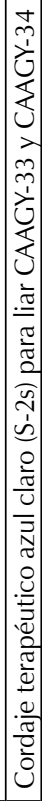 & 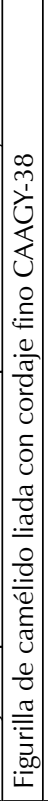 & 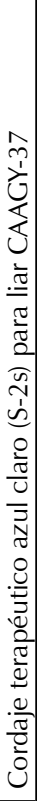 & 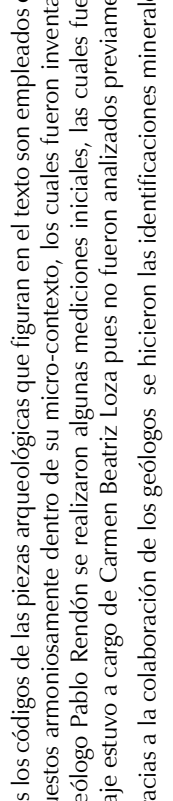 \\
\hline 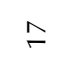 & $\stackrel{\infty}{-}$ & $\stackrel{\sigma}{\longrightarrow}$ & ح & $\bar{v}$ & ปี & 1 & $\stackrel{ \pm}{\sim}$ & $\stackrel{\sim}{\sim}$ & T & & $\stackrel{9}{9}$ & ○ & त & 8 & n & n & & & l & $\hat{m}$ & $\stackrel{\infty}{m}$ & 8 \\
\hline
\end{tabular}


de sujeción. Este último fue fabricado uniendo dos tipos de elementos distintos que juntos suman un total de $133 \mathrm{~cm}$ de largo. El primer cordaje (de $68 \mathrm{~cm}$ de largo) se conecta gracias a un nudo simple con una cinta tejida (de $2 \mathrm{~cm}$ de ancho y $65 \mathrm{~cm}$ de largo) adornada con diseños de indudable filiación tiwanakota. Aclaramos que esta conexión no fue mencionada, ni identificada en los anteriores análisis porque la cinta se torció por el uso, de tal manera que a primera vista parecía un solo cordaje (fig. 1).

\section{2. 3. Los objetos de un equipo inhalatorio incompleto}

La tableta para inhalación de polvos psicoactivos [CAAGY-12] protegida y resguardada en su integridad por un doble cuero protector. El primero, es una pierna de la taruca que cubre el estuche [CAAGY-10], mientras que el estuche es del cuero curtido del mismo animal y del cual se desprende un lazo (de $176 \mathrm{~cm}$ de largo) del mismo material para asegurar y atar la tableta. Destacamos que el estuche, debido a su uso y al extenso tiempo que la contuvo, adoptó la forma del relieve de la misma [CAAGY-11]. Aunque parezca obvio, conviene subrayar que la tableta es hasta el presente una de las más grandes identificadas de filiación Tiwanaku. Está asociada a varios estuches para guardar los polvos psicoactivos. Las características básicas son:

- Un estuche de cuero de jaguar de tamaño medio con larga flecadura de 26,5 cm de largo de cuero de taruca; ambos están unidos por costuras con hilos de pelo de alpaca. Se debe destacar que la parte destinada a ser reservorio tiene una dimensión de $11 \mathrm{~cm}$ de largo máximo, superando esa dimensión la flecadura de taruca con 15,5 cm de longitud máxima [CAAGY-7].

- Otro cuero de jaguar, en forma de «estuche» de menor tamaño que el señalado previamente, pues solo mide 10,84 cm de largo [CAAGY-08]. Albergaba una cucharillita de hueso de clara filiación Tiwanaku por la iconografía en su anverso y reverso, como lo apreciaremos más adelante. A lo largo del mango tiene detalles de zigzag, destacándose en el reverso un ícono similar a un rostro antropomorfo (fig. 1). Además, en la parte superior del mango, tiene tallada una cabeza de ave de rapiña (probablemente un águila) [CAAGY-09]. La cucharilla —untada con grasa de camélido- debió servir para extraer y medir los polvos usados durante rituales para la curación de enfermedades/ padecimientos/infortunios. Vuelvo a repetir que se ha verificado la ausencia de tubos inhalatorios en el conjunto de elementos asociados a la tableta de Pallqa. Durante la prospección y excavación de 1998, no se ubicó ninguno de estos tubos en los abrigos rocosos identificados e inspeccionados por la UNAR (AUNAR, 1998e).

- Un fragmento textil policromo antropomorfo [CAAGY-06]. Este elemento fue colocado dentro de la bolsa textil tipo saquillo [CAAGY-09] y descrito más adelante.

\section{2. 4. Los cueros-envolventes intermedios de múltiple función}

Si se decide estudiar el contenido del watasqa de Pallqa en forma independiente cada uno de los objetos y elementos que guarda, es decir desgajados de su contexto cultural pierden sentido de su valor y significación. En la medida en que cada elemento cobra sentido en el seno de su sistema global y ese sentido es adquirido socioculturalmente. En consecuencia, son las asociaciones de la diversidad de elementos guardados y organizados jerárquicamente los que le confieren significación. Por ello, las uniones de elementos han sido identificadas, en este texto, como «preparados curativos», lo que incluye no solo el contenido guardado en los cueros, sino el continente. Vale decir que el preparado tiene un papel activo que adopta en el proceso curativo ritual, tal como lo demostraremos después. 


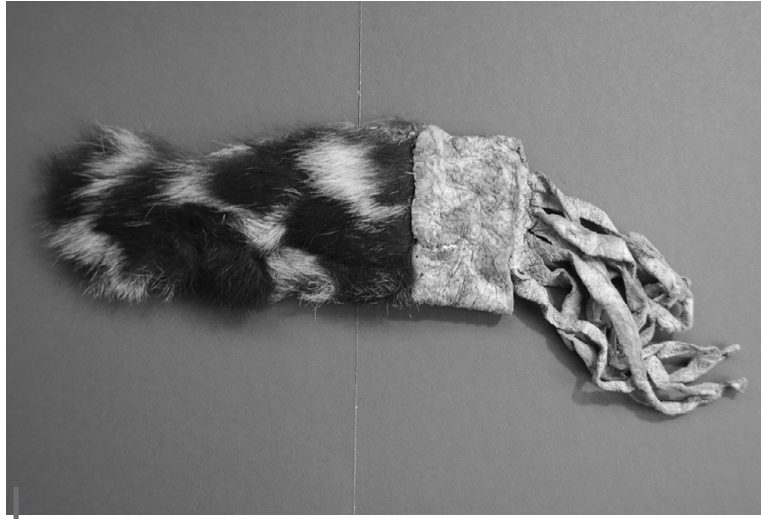

Figura 1 - Fotografía de un estuche de cuero de jaguar para guardar la cuchilla de hueso
Hemos reconocido que en el sistema de almacenamiento se añade la existencia de dos categorías más de cueroscontenedores con preparados, considerando como variables la dimensión y su posible uso. Los cueros-contenedores intermedios guardan los cueroscontenedores pequeños que son portadores de preparados y funcionaron para llamar a los «espíritus» de los animales y minerales que contienen. Mientras que los cueroscontenedores diminutos, los

últimos de la cadena del almacenamiento, guardan gran variedad de elementos orgánicos e inorgánicos de diferentes tamaños y formas.

\section{LOS PREPARADOS CURATIVOS, UNA IDENTIFICACIÓN DE SUS MICRO-CONTEXTOS}

\section{1. Tres clases de remedios/cueros-contenedores intervenían en los preparados curativos}

Argumentamos que todos los remedios/cueros-contenedores, sea cual fuere su dimensión, también confieren sentido simbólico a su contenido, apoyándonos en los testimonios de los expertos indígenas entrevistados. A lo largo de este texto entendemos a estos y su contenido como preparados curativos por las propiedades medicinales que les atribuyen. Al proponer esta terminología queremos connotar que los cueros intervienen en ese proceso, tal como se lo verifica a nivel etnográfico. Estos datos, a su vez, nos sirven de fundamento para sostener que no estamos en presencia de simples y comunes «bolsitas», «estuches» o «saquitos» y menos aún ante recipientes homogéneos destinados únicamente a guardar o conservar algo. En otras palabras las categorías mencionadas son insuficientes. ¿A partir de que pautas nosotros suponemos que estas categorías vehiculan una visión unívoca, negando una diversidad de empleos y significaciones en el ámbito de la práctica curativa andina? Una respuesta se esboza si nosotros establecemos un paralelo con los empleos que los kallawaya y k'awayu que hacen de cueros, de acuerdo a reglas, códigos y terminología técnica muy precisa. Se establecieron tres modalidades diferenciadas.

La primera modalidad es la q'ipichaska, caracterizada por envolver ciertos objetos (como los minerales) en el cuero, sin que necesariamente porten un cordaje fino para sujetarlos; en general están en proceso de elaboración y se les podrá añadir nuevos elementos. La segunda modalidad es el watasqa, se distingue de la anterior porque los elementos pueden estar herméticamente obturados en el cuero y asegurados con cordajes de sujeción o cordajes terapéuticos que terminan en un amarre. Un ejemplo, es el propio watasqa de Pallqa que estamos analizando. La tercera modalidad, es la waqichaska porque los remedios/cueros-contenedores menores y diminutos pueden desempeñar un papel activo en el preparado al cobijar elementos que son asegurados con cordajes terapéuticos (para mayores detalles véase el acápite 2. 2.). 
Sea cual fuere la modalidad, estas tres fueron utilizadas en las curaciones en el periodo Tiwanaku y continúan vigentes de acuerdo a las indicaciones de los kallawaya Walter Alvarez Quispe y Max Chura, quienes nos ofrecieron una explicación a partir de su propia observación del atado de Pallqa y la tradición recibida de sus antepasados kallawaya (entrevistas 4 y 5). Dos de ellas son claramente visibles, como sigue:

\section{1. 1. Las modalidades y funcionalidad de los cueros-envolventes intermedios y su continente}

Partiendo de esta concepción, hemos distinguido la existencia de un cuero contenedor intermedio de vizcacha que encierra otros cinco cueros-contenedores diminutos también de vizcacha. Todos ellos, responden a la tipología anteriormente descrita:

a. Un wakichisqa de vizcacha cuyo cuero-contenedor es de gran interés porque encierra una sucesión diferenciada de otros cueros-contenedores de menor dimensión. Estos últimos pueden ser identificados, según la tipología indígena aún utilizada en el presente por los expertos indígenas.

b. Un wakichisqa que a su vez encierra, siguiendo el mismo principio, un cuero-envolvente/ contenedor pequeño de vizcacha, el cual albergaba preparados para las enfermedades/ padecimientos/infortunios, lo siguiente:

- Un watasqa miniatura fabricado en cuero de taruca liado con fino cordaje azul claro para asegurar un pequeño cuarzo.

- Un watasqa miniatura, también fabricado en cuero de taruca liado con cordaje fino azul claro para asegurar ápices de pirita.

- Un watasqa miniatura de cuero de taruca liado con fino cordaje azul oscuro para asegurar cuarzo.

- Un watasqa armado a partir de una punta de proyectil bifacial del tipo hoja de laurel (con una pequeña fractura en la punta), elaborada en arenita de cuarzo endurecida. Este proyectil lleva envuelto y adherido, en su parte media, plumones de ave (posiblemente picaflor por su colorido y tamaño) con un cordaje zurdo teñido de color azul oscuro que envuelve la punta dos veces y la sujeta con un nudo simple. La punta es de hace 4000 años a.J.C. (Holoceno), asociada a los cazadores de llamas de la llamada fase Puri-PicaTolar. Es decir, que el propietario del atado reutiliza objetos que son 3000 años anteriores al periodo que le tocó vivir para ejercer su oficio. Tal como lo hacen los k'awayu hoy en día reutilizando puntas de flecha buscando protección.

- Un cuarzo asociado al watasqa arriba señalado como parte integrante del wakichisqa.

c. Un wakichisqa cobijado en el cuero-contenedor de vizcacha que contiene un watasqa organizada de la siguiente manera:

- Un watasqa formado por una base de vellón de lana de vicuña para proteger y cobijar un cálculo de camélido y en una de sus protuberancias se amarra un cordaje fino azul claro.

d. Un wakichisqa cuero-contenedor pequeño de vizcacha que guarda tres watasqa en su seno:

- Un watasqa formada a partir de una obsidiana liada con un fino cordaje azul claro.

- Un watasqa formado sobre una base de vellón de lana de vicuña en el cual se colocó un canto rodado finamente cubierto y seguramente asegurado con el cordaje fino azul claro.

- Un watasqa conformado por una figurilla de camélido cuyo cuello está unido con un cordaje fino azul claro. 
Hasta acá, hemos efectuado una presentación general completa del contenido del atado de Pallqa. Como se puede apreciar, resolvimos el primer problema que nos ha ocupado: distinguir la especificidad del atado dentro de la terminología andina como un watasqa y, confirmar que esta modalidad de preparado esta presente en diversos niveles de la organización de los remedios, particularmente en el nivel de los preparados diminutos. Al mismo tiempo, la diversidad de objetos y sus asociaciones nos obligan a considerar los micro-contextos en los que hace su aparición. Este recorrido aunado a la información ofrecida por los expertos indígenas entrevistados permitirá aproximarnos, de manera más segura, a las posibles significaciones del atado y los objetos que incluye. Una entrada es el análisis de los cordajes.

\section{2. Las seis variantes de finos cordajes como ayuda mnemónico-terapéutica}

El cordaje ha sido uno de los elementos más descuidados en los análisis anteriores, a pesar de constituir un elemento clave para la comprensión de los preparados. Nuestro análisis ha establecido las garantías de una tipología importante y novedosa. En primer lugar, los cordajes finos fueron elaborados por el propietario del atado, quién tomó fibra animal para formar dos hilos que al ordenarlos los torció a la izquierda (torsión en «S») para fines terapéuticos (o sea, S-2s) porque connotan sentido ritual y curativo, pues participan en el cambio de dirección de las enfermedades/padecimientos/infortunios. Debido a esa característica son denominados Iluqu'i q'aytu. Luego, hemos establecido una diferenciación interna en los cordajes a partir de cuatro tipos de ovillos terapéuticos distintos para liar y amarrar los preparados. Cada uno de ellos varía en su grosor, aunque predomina la tonalidad azul. Finalmente, determinamos que las costuras de los estuches de jaguar fueron cocidas con lluqu'i q'aytu de vicuña. Este conjunto de resultados de análisis son la garantía para autorizarnos a negar la existencia de un solo ovillo como se había planteado anteriormente (Capriles, 2002: 44). En consecuencia podemos profundizar en la función operativa de seis tipos de cordajes finos presentes en el atado.

Uno de los empleos del cordaje fue la sujeción y codificación de información en los preparados watasqa de Pallqa. Por ejemplo, se evidencia en el preparado de la lasca que brindaría protección a su poseedor. En efecto, se verifica que está armado a partir de una punta de proyectil donde se sujetó plumas de picaflor con un hilo zurdo azul, el cual da dos vueltas para concluir con un nudo simple. Es probable que fuera construido para uso exclusivo del religioso/médico debido al montaje de un elemento con punta esgrimido para alejar a los malos espíritus. De hecho, el picaflor participa en la curación de ataques (APCBL, entrevistas 7, 21; Girault, 1987: 498).

Los cordajes de vicuña sujetan los cueros de vizcacha que contienen minerales. Esta asociación no es casual. La vicuña — según los k'awayu — sería la encargada de transportar, por la noche, los minerales para los dioses-antepasados en virtud de ser uno de los «animales preferidos de los espíritus ancestrales» (APCBL, entrevista 14; Girault, 1987: 516). Así, existiría una lógica asociación de los significados simbólicos utilizados por el religioso/ médico... Lo curioso es que en los preparados curativos del religioso/médico todos los hilos, sean de vicuña o de alpaca están teñidos de azul. Esta situación nos interroga acerca de la función y significación que pudieron haber tenido. Sobre todo porque no es la primera vez que aparecen en la evidencia arqueológica andina. Por ejemplo, según un informe de los trabajos de excavación en el valle bajo de Osmore (Moquegua, sur del Perú) se encontraron: 
«hilos muy finos teñidos de azul por lo general junto a sandalias de cuero en miniatura y para amarrar pequeñas patitas de cuy, lo que sugiere que este tipo de hilo tuvo una función muy significativa» en el periodo intermedio tardío (Clark et al., 1999: 112).

Sabemos por la información recogida en terreno potosino que los Iluq'i q'aytu azules continúan siendo empleados y son ampliamente valorados en ciertos grupos indígenas del altiplano y valles bolivianos. Se reconocen, al menos, dos atributos tradicionales que se han mantenido vigentes.

- Transmiten la fuerza a sus portadores. Por ejemplo, los varones que participan en las luchas ceremoniales del T'inku de Macha (uno de los más sangrientos en el municipio de Colquechaca, de la provincia Chayanta, Potosí) se colocan secretamente en sus cascos los lluq'i q'aytu azules para tener más poderío y vencer al guerrero contrincante.

- Invierten el curso de ciertas enfermedades/padecimientos/infortunios. Es el caso de los K'awayu que los emplean en las curaciones simbólicas que aquejan las dolencias de amor, según una experta —Paulina Chucamani- los médicos indígenas manejan los Iluq'i q'aytu azules en ciertas ceremonias de cambiaq para invertir la suerte. Pero ella advierte que se prescindió de su venta debido a la incorporación de velas de color en la ritualidad andina, a raíz de la influencia brasileña y su pujante industria de objetos simbólicos que invaden acelerada y eficazmente el mercado boliviano. De esa manera, las velas estarían en proceso de desplazarlos, aunque sin lograr eliminar todavía a los hilos de oveja blanca y negra de los que se piensa contrarrestan las maldiciones o los hilos blancos para la buena salud (APCBL, entrevistas 9 y 16). La afirmación de Paulina Chucamani nos condujo a una búsqueda minuciosa en los mercados de objetos rituales de diversas ciudades bolivianas, llegando ubicar lluq'i q'aytu azules en el Mercado Vicuñas de la ciudad de Potosí. En efecto, otra experta K'awayu - Paulina Saravia Quispe — todavía los expone y vende en su puesto alrededor de 20 tipos diferenciados de Iluq'i q'aytu (APCBL, entrevista 17). En otros términos, se conserva esta tradición, por lo menos desde la época de Tiwanaku, debido a que el gran mercado potosino está marcado por un vigoroso ritualismo andino en razón de la existencia de las afamadas minas argentíferas que dinamizan su espacio socioeconómico y que requieren de la atención privilegiada de los médicos y ritualistas andinos. Pero también se explica la continuidad por la vitalidad de los ayllu potosinos y sus miembros que siguen conservando tradiciones culturales muy antiguas que intervienen en el proceso de la salud/enfermedad/atención, a pesar de la evangelización católica y de la temible amenaza extirpadora las sectas evangélicas que aceleran la destrucción cultural andina, dividiendo a sus pobladores y condenando, estigmatizando y satanizando a quiénes permanecen fieles a sus tradiciones curativas y sus prácticas religiosas de raigambre andinas.

\section{3. Los preparados curativos, una identificación de sus funciones}

La identificación del contenido no es suficiente en el análisis, es necesario profundizar en otras dimensiones del mismo, retomando la clasificación básica de los animales de acuerdo a las etnocategorías utilizadas por los andinos para vislumbrar su importancia y entrever su significado (Flores Ochoa, 1977: 231; Gutiérrez Usillos, 1989). Se han identificado, que el religioso/médico utiliza dos grandes categorías de animales en la elaboración de sus cueros de embalaje (envolventes y contenedores) y son aquellos que gozan de una situación privilegiada en la cosmovisión andina. En primer lugar, los animales silvestres, denominados salqa en quechua, como por ejemplo: la vizcacha, jaguar, vicuña y taruca. 
Por otro lado, utiliza animales domesticados, uywa en quechua, de los cuales extrae su fibra con diferentes fines. Así, la distribución estadística muestra que sobre un total de 25 objetos, se verifica una clara tendencia a privilegiar a los salqa con un 57,69\%. No menos importante son los domesticados con un $26,62 \%$, siendo el 7,69 \% de sus objetos combinación de salqa con uywa.

Esta descripción constituye la base para sostener que la elección de los materiales empleados por el religioso/médico no ha sido hecha al azar, sino que ha habido relación directa con aquellos animales silvestres o salqa que pertenecían a los rebaños de las deidadesantepasados (denominados Machulas o Apus), en consecuencia comportan un carácter sagrado. En esta misma dirección se puede entender su preferencia por los animales salqa y no por la categoría uywa que son domesticados y «dados en préstamo a los pastores», por ejemplo la alpaca que es una entrega de la «Señora del tiempo» y del espacio Pachamama (Flores Ochoa, 1977: 230).

La primacía del cuero es altamente significativa, se conoce sus atributos en todo el proceso de Tiwanaku y su uso pervive hasta el presente. Por ejemplo, tanto los K'awayu como los kallawaya, por lo menos hasta la segunda mitad del siglo XX, han destinado los cueros para preservar objetos simbólicos y guardar sus medicamentos. Sin embargo, esta tradición está desapareciendo aceleradamente debido a la sustitución por nuevos y poderosos materiales de gran aceptación popular: telas, papel y plástico.

En efecto, fueron los kallawaya los que reemplazaron los cueros, paulatinamente, por casimires y tela burda de algodón (APCBL: entrevistas 5, 7, 8, 11, 12, 14 y 15). Justamente, en la colección de instrumental antiguo de los antepasados Kallawaya de Walter Alvarez Quispe, tuvimos la oportunidad de examinar una alforjita de casimir — perteneciente a sus antepasados herbolarios - con muchos bolsillos pequeños, la cual servía para ocultar objetos simbólicos de fácil transporte: un amuleto pequeño que representaba la pequeña mano del médico y una bolsita menuda de tela para conservar plantas secas molidas amarrada con hilo de color rojo (S-2s) y otra bolsita para minerales3. Las bolsas plásticas han terminado por imponerse entre la mayoría de la población boliviana, incluidos los expertos indígenas. Por citar un caso, actualmente los k'awayu solo guardan sus medicinas en coloridas bolsas plásticas y en algunos casos recipientes del mismo material -introducidas por la cooperación internacional en nombre de la higiene y el progresohabiendo dejado reservado el uso de los cueros-contenedores para guardar centenares de medicinas pertenecientes al Santo Patrón de los k'awayu, quien tiene un jampi q’ipi como cualquier otro miembro del grupo étnico especializado en la preparación, intercambio y venta de objetos simbólicos y medicinales (APCBL: entrevistas 13 y 21). Los cueros solo son repartidos como objetos simbólicos medicinales y entregados a los solicitantes y pacientes en papel (generalmente periódico) protegido por una bolsa plástica.

\section{3. 1. Los remedios, su combinación en los preparados}

Una vez descritos los materiales de acuerdo a diversos sistemas de clasificación, nos hallamos en condiciones de interrogarnos acerca de los preparados medicinales en sí mismos; para ello asumimos que el tipo de cuero juega un papel de «marcador» porque señala significados. En general, los elementos se inscriben en una configuración que podemos

3 Estos objetos fueron expuestos durante la exhibición itinerante «Kallawaya. Cultura, ciencia y memoria» organizada por Carmen Beatriz Loza junto a los kallawaya en el Espacio Simón I. Patiño (La Paz, Cochabamba, Santa Cruz de la Sierra y Sucre) entre marzo y octubre de 2005. < http: ea.el-nuevodia.com/2005/04-Abril/01Abril2005/Revista Escape/Marzo/esc050325b.html> (Consultado el 24 de marzo de 2006). 
describir en un primer trazo, a partir de un esquema alrededor de dos polos; a saber, los preparados para prevenir y aquellos empleados para curar.

Por ejemplo, la taruca, por su condición de salqa, tiene varios atributos e interviene en curaciones rituales específicas en el mundo kallawaya, además el pelo es empleado como un gran protector contra el «mal viento», al cual se le atribuye la parálisis facial (APCBL: entrevistas 7 y 14). Estos usos contemporáneos nos ofrecen pistas para comprender que las capas constitutivas del armazón del watasqa de Pallqa estarían indicando claramente la pertenencia a un individuo relacionado con el mundo simbólico y ritual de su tiempo. Mientras que la vizcacha — también debido a su condición de salqa — está relacionada con las medicaciones y la prosperidad. Por esa razón, actualmente los mineros potosinos ofrendan durante la «curación de la mina» los fetos de ese animal silvestre para descubrir muchas vetas de mineral y alcanzar la prosperidad demandada a las deidades (APCBL: entrevistas 10, 13 y 14). Tal afirmación, lograda gracias a la observación participante en el acto de la «curación de una mina», es de suma importancia para nuestros propósitos porque confirma la coherencia en la asociación realizada por el religioso/médico, quien de manera sistemática conserva los minerales en cueros-contenedores de vizcacha como lo ilustra el cuadro 1.

La suerte está representada en el cuero de jaguar porque está mandado para morar en los lugares sagrados (Rösing, 1992: 244). Por esa razón, es imprescindible hasta el momento actual en los preparados de ofrendas blancas de protección y bienestar, pero también para las «ofrendas negras» destinadas a transformaciones en el curso de la vida (APCBL: entrevistas 5 y 7). El religioso/médico lo utilizó para elaborar sus cueros-contenedores y el estuche donde enfundaba la cucharilla para los diversos polvos y psicotrópicos.

El religioso/médico produce medicamentos que pueden ser empleados para curar enfermedades físicas y mentales, siendo algunos de sus ingredientes, imprescindibles en las curaciones simbólicas. Es el caso de los cálculos de los camélidos denominados en tiempos coloniales por los españoles «piedra bezoar»(De Losa, 1983 [1780]: 6365). Es conocido en quechua como karwa jayintilla, según el testimonio de los actuales k'awayus. La guardan delicadamente envuelta en vellones de lana de alpaca en tanto es muy apreciada no solo por la dificultad de conseguirla sino también porque es portadora de los «espíritus» del animal. Ese valor que le otorgan es muy antiguo, todavía en tiempos coloniales lo consideraban «gran remedio» y según los observadores de la época los indígenas la utilizaban:

«a tiempo que iban a pelear, diciendo que les da valor y fuerzas y asimismo apartaba de ellos todo temor y miedo, alegrándoles el corazón» (De Losa, 1983 [1780]: 63).

En la actualidad, los Kallawaya la utilizan por un lado, en infusión como si fuera chocolate porque ayuda a la digestión y mejora los dolores estomacales (ACBL, entrevista 5). Por el otro, interviene en las curaciones de susto, una de las patologías andinas más importantes (APCBL: entrevistas 6,18, 21). Sin embargo, en tiempos coloniales sus funciones eran más amplias, servían de:

«antídotos contra todo veneno, tabardillo, melancolía, pasiones del corazón, contra calentura pestilente o pútrida, contra el aire» (De Losa, 1983 [1780]: 63; APGL).

Todo parece indicar que la karwa jayintilla liada con lluq'i q'aytu fue utilizada con propósitos de curación simbólica.

En concreto, en los preparados curativos del religioso/médico apreciamos elementos de distinta naturaleza: animal, vegetal y mineral, los cuales pueden servir para medicaciones de patologías culturales o enfermedades físicas reconocidas. Y, al parecer, la población 
de Chacapa y Challana — donde pertenece el sitio de Pallqa - desde antaño valoró y aprovechó los tres reinos, manteniéndose la tradición todavía hasta inicios de la Colonia. En efecto, los españoles notaron que los indígenas, todavía a fines del siglo XVI, tenían un gran apego al rescate de pieles y de guacamayas, «plumas de pájaros muy pintados» (Dávila de Cangas \& de Otazú, (1991 [1568-1569/1570]: 286r). No obstante, en esa época, los mismos españoles minimizaban el interés de los indígenas por esas «cosas superfluas». En consecuencia, no imaginaban que servirían para elaborar preparados para curaciones simbólicas, su propia defensa y ostentar sus símbolos de poder. Más bien, creían que el empleo de «gran plumería» y pieles conllevaba sencillamente fines ornamentales y que era equivalente a la afición por lucir camisetas, manillas, brazaletes y anillos de oro y plata (Dávila de Cangas \& de Otazú, (1991 [1568-1569/1570]: 268r., 648v.).

\section{EL AJUAR INHALATORIO, UNA PRESENTACIÓN DE SU MICRO CONTEXTO}

\section{1. Una tableta ceremonial en madera}

Hasta el momento nos hemos dedicado a detallar básicamente los preparados curativos. Se hallan también algunos elementos básicos del ajuar inhalatorio que han debido intervenir en las prácticas del religioso/ médico. En una primera observación destaca la tableta de madera de Pallqa que es, desde un punto de vista arqueológico, una pieza distintiva por el contexto no precisamente funerario en que fue hallada, su gran tamaño y la funda completa que la conserva y resguarda (fig. 2).

Al parecer, la tableta ha sido probablemente tallada en madera chonta, considerando el peso, sonido y textura de la misma. Comparándola con otras, Ilama la atención su gran tamaño: una longitud máxima de $24,6 \mathrm{~cm}$ y una anchura máxima de $9,1 \mathrm{~cm}$, siendo su grosor de 1,23 cm. Baste señalar que en San Pedro de Atacama, norte de Chile -Quitor 5, tumba 20212034 de acuerdo a Le Paige- existe una tableta que tiene de largo de $27,5 \mathrm{~cm}$ y aproximadamente $9 \mathrm{~cm}$ de ancho. Sin embargo, la tradición de tabletas grandes no es privativa de tiempos prehispánicos, sino que pervive en algunos lugares del Amazonas hasta el tardío siglo XIX, sobre todo en el Maué. Tabletas de $37 \mathrm{~cm}$ de largo, llegando incluso a $45 \mathrm{~cm}$, pertenecen a este último rango ${ }^{4}$.

Adhiriéndonos al sistema de clasificación propuesta por el arqueólogo Constantino Manuel Torres, a partir del
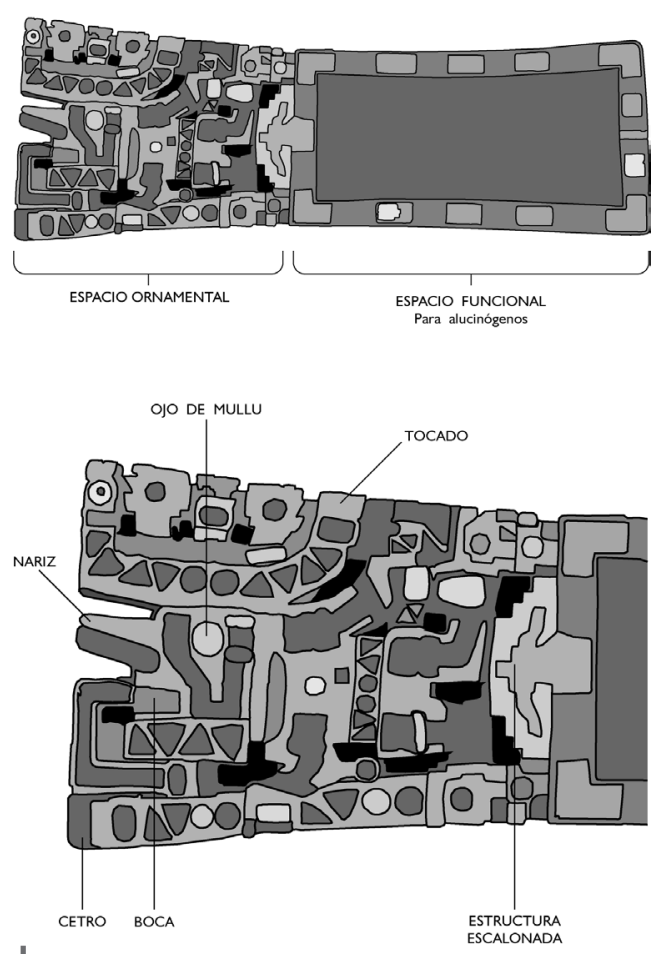

Figura 2 - Tableta ceremonial de Pallqa

4 La primera es la de Munich y la segunda de Coimbra. Constantino Manuel Torres. 2005. Comunicación a Carmen Beatriz Loza 27 de abril. 
análisis de 713 tabletas en el cual se sustenta la tipificación de objetos provenientes de sitios dispersos en el actual norte de Chile, la tableta de Pallqa morfológicamente, pertenece al tipo B y sería «al menos dos veces más grande que las tabletas Tiwanaku encontradas en San Pedro de Atacama» (AUNAR, 1999c). Tiene extensiones planiformes con incisiones lineales, además del tallado en relieve (Torres, 2001; Rendón, 2000: 90). Morfológicamente tiene dos espacios bien diferenciados y complementarios compartidos por gran cantidad de objetos de este tipo: el espacio funcional, por una cavidad central plana que hace de recipiente subrectangular tallado y que sirve de bandeja para preparar polvos de vegetales y mezclarlos o simplemente para inhalarlos. En este caso, todo este recipiente fue utilizado porque la cavidad mutó de coloración, posiblemente debido a que en ella se combinan los ingredientes para preparados psicotrópicos.

Originalmente la tableta ostentaba incrustaciones de metales, piedras semipreciosas y de conchas en el borde así como en diferentes lugares de la representación íconográfica, pero actualmente varias incrustaciones lucen huecas, debido, en parte, a las intervenciones de su descubridor, Francisco Pilco. El filo está decorado con una serie de figuras geométricas en bajorrelieve e incrustaciones de concha marina rojiza o mullu5, tal cual la nombran en el mundo andino. En los extremos se aprecian cuatro elementos en forma de L. Sobre el borde, entre las figuras, destaca un rectángulo; mientras que en los costados otros tres rectángulos se encuentran intercalados a distancias regulares; a su vez, dispuesto en el costado izquierdo un rectángulo ostenta una incrustación de mullu. Debajo del borde hay dos triángulos distanciados por un pequeño surco que abarca el espesor total del linde decorado, uno de dichos rectángulos está ornado por un mullu.

\section{1. 1. El personaje de Pallqa en la tableta}

En la tableta se advierte un personaje masculino, ricamente ataviado y ornado con emblemas que ofrece una puesta en escena de filiación claramente Tiwanaku. El personaje de Pallqa — denominación empleada a lo largo del texto— está encima de una estructura escalonada de tres niveles, flanqueada por dos cabezas de puma con un ojo de mullu. Sobre esta estructura se apoya el cuerpo varonil haciendo ademán de inclinar su rodilla derecha, mientras que su peso descansa sobre la siniestra. Al mismo tiempo, sus brazos se extienden ligeramente, el derecho empuña un cetro con accesorios de compleja simbología, que atenderemos más adelante, en tanto que el brazo izquierdo mira hacia abajo en dirección a la estructura que sostiene otro tipo de elementos, los cuales serán descritos luego.

Los primeros emblemas de dignidad descollan en su cabeza. En efecto, un amplio tocado «coronado» resalta el rostro del personaje. Sobresale una testa en forma de herradura, claramente visible de frente, con una sucesión de íconos. Viendo de derecha a izquierda se distingue en un extremo el símbolo del fuego, seguido de cuatro triángulos, dos círculos y cuatro triángulos nuevamente; en sentido opuesto se yergue una cabeza de puma, cuyo ojo es de malaquita. Al centro un símbolo de fuego, igualmente con una incrustación de malaquita, va flaqueado por dos cabezas trofeo, una de las cuales presenta un ojo con incrustación de turquesa y concha. Por hallarse en plano su rostro de perfil, se halla inclinado hacia arriba, distinguiéndose su prominente nariz. De su boca entreabierta sale un símbolo en forma de bastón invertido, a modo de lengua, el cual lleva engastado en la parte inicial un pequeño mullu; al final sobresale el símbolo del fuego. Contiguo al mentón y a la mejilla, luce una gargantilla con cuatro triángulos que intercalan su posición, uno de ellos, el más cercano al cuerpo, lleva taraceada una turquesa.

5 «Mullo: Piedra, o hueso colorado como coral con que hacen gargantillas y también usan las hechizeros» (Bertonio 1989 [1612]: 227). 
El personaje de Pallqa, deja ver solo en uno de los ojos la córnea con lagrimal, en cuyo interior se vislumbra la niña donde se ha engastado un círculo grande de mullu. Todo indica, siguiendo los cánones andinos, que nos hallamos frente a un hermoso varón de ojo colorado. Este atributo de belleza, permeó varios siglos, al extremo que los lupaqas del lago Titicaca recurrieron al vocablo mullu nayrani para designar a los hombres bellos. Este término todavía era utilizado cuando regía la Corona española (Bertonio, 1989 [1612]: 227].

El pecho del personaje que describimos está en posición frontal y ataviado por una vestimenta asegurada en la cintura por una tradicional faja abdominal requerida en ciertas solemnidades y por eso mismo engalanada por una sucesión de figuras geométricas: dos triángulos a cada costado y en medio dos círculos. Cobra relieve, por estar situada en el centro de la representación pectoral, una cabeza trofeo vista de perfil, donde uno de sus ojos está damasquinado de turquesa. En cualquier caso, la íconografía del personaje de Pallqa no agota aquí la complejidad de la puesta en escena, pues se observa que las insignias que lucen los brazos, sobreponen signos distintivos. En tanto en el brazo derecho, despuntan dos elementos decorativos, el primero consistente en un símbolo de fuego que se extiende sobre todo en la vestimenta hasta llegar alcanzar al pecho, en el segundo se observa un objeto circular que cuelga del codo como formando parte de la indumentaria del personaje, el mismo que se encuentra doblado en ademán de sostener un cetro. Uno de los símbolos de poder que merece ser descrita es el cetro, por el modo como se sobreponen los signos en esta insignia. La parte superior del cetro luce el símbolo del fuego, seguido de dos triángulos y dos círculos uno exento de mullu. En la mano se colocó una incrustación de mullu que simboliza las uñas de los dedos. Luego, se suceden otros dos triángulos y un círculo con mullu y nuevamente otro círculo en bajorrelieve para culminar en una cabeza trofeo en ángulo de perfil.

El brazo izquierdo, siguiendo el mismo principio, presenta un símbolo de fuego que se inicia en la muñeca para culminar en el pecho. La mano con uñas engastadas de bronce - metal denominado en aymara, Ilacsa-. Esta mano sostiene un hacha, cuyo mango va ornado por cuatro triángulos, los cuales se oponen en pares. Al mismo tiempo, sujeta por los cabellos una cabeza trofeo. El bronce que embellece la tableta de Pallqa engalana los dedos de la mano izquierda. Dentro de la ideología andina, el bronce, en aymara Ilacsa, era un metal que infundía temor a los rivales. Conforme Gonzáles de Holguín, se lo utilizaba para:

«pasmar a otro, hacerle turbar de miedo o cortarle, o desmayarle, o helarle la sangre como el que ve un león» (Gonzáles de Holguín, 1952 [1608]).

Lo que significa que el personaje sería un decapitador, ahuyentador de aquello que le causaba molestia o aflicción. En la puesta en escena, sin embargo, aparece algo digno de consideración, es el simbolismo de las incrustaciones en los dedos: en los de la mano derecha mullu y en los dedos de la mano izquierda, bronce. De esa manera, el gesto de nuestro personaje estaría articulando elementos de poder: mullu y bronce presentes como una asociación importante y común en la cultura material de Tiwanaku (Cf. Kosok, 1965: 39, Paulsen, 1974: 597). Basándonos en la descripción anterior, obtenemos conclusión ilustrada en la figura 3. A partir del mismo, vemos cómo el personaje de Pallqa encarna la dignidad, bravura y belleza.

\section{1. 2. De los textiles menos abundantes}

Como lo señalamos, un fragmento textil se hallaba colocado en la bolsa que contenía la mayoría de los implementos del médico/ritualista. El fragmento de doble cara aparentemente perteneció a un tipo de bolsita con un tiro de cuero. El mismo da cuenta de un personaje 
portador de una camiseta ritual denominada en quechua unku. Su rostro amarillo de perfil sobresale en un fondo azul claro; de ahí que se aprecie un solo ojo bipartito, que exhibe una porción mínima de color negro y el restante blanco. Del ojo se desprende un lacrimal dividido en dos partes: la más cercana de la boca y la nariz representa un felino con ojo bipartito que mira hacia abajo. En el otro extremo, muy cerca de la oreja se observa un diseño de plumas. Sobre la oreja se perciben dos líneas, una blanca y la otra roja, que acaban en un diseño geométrico, probablemente se trate de cabellos. La nariz destaca en el rostro por su albura, debajo de ella se aprecia la boca que enseña los dientes.

Adorna la parte superior de la cabeza una corona blanca en cuyos extremos lleva dos círculos concéntricos rojos y al centro amarillo. Además de un símbolo de fuego flanqueado por dos cabezas de pescado con ojos bipartitos. Por detrás de la oreja, se observa diseños en forma de bastón invertido en tres matices: amarillo, rojo y blanco; esta figura recuerda un cetro. Este diseño vertical es seguido de otros dos compuestos de formas geométricas, tanto rectángulos como triángulos, de difícil definición. Por delante del rostro aparecen dos diseños en posición vertical y uno debajo del otro, similares al número seis. El primero es rojo y el segundo blanco con un punto amarillo y azul. Seguidamente se observa una sucesión de signos y figuras geométricas, las cuales posiblemente representen un cetro, continúan varias líneas de color azul, rojo y blanco. En la parte inferior del textil se aprecian algunos diseños geométricos incompletos y un ojo bipartito en un fondo rojo, que reposa sobre un espacio azul. El deterioro del textil impide una adecuada identificación íconográfica (APCBL: entrevista 19).

\section{CONCLUSIÓN}

De este modo y finalmente, el cruce de miradas en torno al atado de Pallqa $y$, las idas y venidas entre temporalidades distintas a través del diálogo con los expertos indígenas nos ha proporcionado una importante base para caracterizar con mayor seguridad su contenido. Esta aproximación cruzada encara en nuestra perspectiva de encuadre, los mecanismos más o menos explícitos de ajustes a las exigencias y limitaciones del objeto de análisis, tal cual se sitúa su producción en la historia. Pero, además establece posibles vinculaciones entre objetos que se utilizan hoy en día que parecen provenir del periodo Tiwanaku, lo cual sugeriría una transposición de siglos. El atado depositado por el religioso/médico fuera de contexto funerario deja establecido que tendría: preparados curativos para enfermedades/ padecimientos/infortunios frecuentes en esa época, implementos del ajuar inhalatorio de substancias psicotrópicas y preparados para su propia protección.

Hemos detectado que la variedad y complejidad de elementos animales y minerales presentes en el ensamblaje de los preparados curativos fue uno de los medios más importantes para expresar la especificidad de las acciones que realizaba en el proceso de la salud/enfermedad/ atención. Y son, justamente las mezclas de varios ingredientes curativos las que facilitaron el trabajo de identificación porque algunos de ellos continúan siendo utilizados en su totalidad por los expertos indígenas. Mientras que en otros casos, solo algunos componentes son reconocidos y participan de las curaciones que se practican hoy en día.

Pretendimos que el religioso/médico cuidó todos y cada uno de los detalles en la confección de su atado de remedios. En ese sentido, afirmamos que él tenía una estructura de pensamiento secuencial muy eficaz y operativa, debido a que se basaba en un modelo cognitivo de organización simbólica. Este modelo otorga una forma particular de ensamblaje y sistema de organización del almacenamiento de acuerdo a una jerarquía de cueros y a una asociación de elementos de distintos materiales destinados 
a la fabricación de remedios que, cautelosamente, tuvo precaución de combinar, tal como actualmente los kallawaya y k'awayu lo siguen haciendo. Justamente, este aspecto que revela continuidad se ha constituido en una pista importante para entender que los diversos tipos de cueros no solo sirvieron para guardar y proteger los elementos, tal como el análisis arqueológico lo había sugerido, simplificando al extremo su funcionalidad. Más bien, el aporte sustantivo de la investigación etnográfica ha radicado en evidenciar, por primera vez, que los remedios/cueros/contenedores intervienen en el proceso curativo porque son portadores de poderes en sí mismos. No en vano, fueron distinguidos en función de las etnocategorías andinas a las que pertenecen: salqa o uywa.

Vale la pena subrayarlo: desechamos la idea según la cual los cueros fueron simples «bolsitas», «estuches» o «saquitos» que no tienen relación unos con otros. En contraste, proponemos una categorización que busca mostrar su multifuncionalidad, dejando establecido las diferencias en las funciones de los cueros mostrando jerarquías y conexiones relacionales. Por esa razón, hemos creído pertinente distinguir en el embalaje entre los cueros/envolventes y los cueros/contenedores, justamente para evidenciar una gradación en la que intervienen aspectos relacionados con la materialidad, por ejemplo considerando criterios acerca del tipo de piel y sus dimensiones. Esto explica que hayamos establecido una taxonomía de los cueros contenedores en mayores, medianos, pequeños y diminutos, todos ellos con funciones médicas en el ámbito de la prevención y la curación.

En esta circunstancia, la manipulación de elementos semejantes a los de Pallqa por parte de los expertos indígenas, ha sido decisivo para distinguir entre las variedades de cueros y proponer la denominación de preparados curativos. Existen tres tipos: watasqa, q'ipichaska y wakichisqa. Esta diferenciación de cada uno de los cueros merecería un análisis comparativo en posteriores estudios con otras evidencias arqueológicas. Este reconocimiento nos permitió determinar que los materiales contenidos en los cueros funcionan conjuntamente con los hilos. Y la totalidad de esos elementos interviene en la significación que conlleva el preparado curativo. Justamente, el análisis de la torción de los hilos nos proporcionó indicaciones inestimables para entender la función curativa de los hilos zurdos o lluq'i $q^{\prime} a y t u$, aspecto que fue descuidado en las investigaciones anteriores debido a que la noción utilizada fue la de «bolsa», y se impuso en el análisis como una categoría válida ocasionando que se desdeñe la funcionalidad de los cordajes y, en consecuencia, negando una significación valiosa

La sabiduría ancestral de kallawaya y k'awayu en el almacenaje en cueros ha sido decisiva para entender estos elementos como totalidades significantes y significativas. Sin sus indicaciones hubiera sido prácticamente imposible su reconocimiento por la imperceptibilidad de sus rasgos y más aún de sus diferencias. Posiblemente a este último y al hecho de que el watasqa de Pallqa se inscribe en un ámbito estrictamente religioso se debe el que su identificación haya estado vedada a los profanos. A más de esto, el acercamiento a los expertos indígenas nos ha permitido superar los análisis excesivamente centrados en los parámetros arqueológicos que han obstaculizado en el pasado una visión más amplia del atado de Pallqa. En este punto, parece pertinente subrayar que la aproximación multidisciplinaria no debe limitarse a los expertos de la ciencia occidental y más bien ahondar en intercambios fructíferos con los expertos indígenas.

La identificación de casi todo el material por los expertos indígenas es una prueba de una relativa continuidad cultural del manejo de preparados curativos. Sin embargo, la búsqueda infructuosa en los mercados de los Iluq'i q'aytu azules de diversas ciudades bolivianas prueba, a su vez, la profunda transformación que se está operando entre los expertos indígenas. Cambios cuyos resultados son imprevisibles y que se encuentran sujetos a las 
actuales políticas de salud integracionistas y pro-biomédicas, pero también a la vitalidad de las prácticas de los religiosos/médicos y al margen de libertad, tolerancia y actividad religiosa que logren conquistar en la sociedad boliviana del siglo XXI.

\section{Agradecimientos}

Agradezco por la autorización de la consulta del material arqueológico a la Unidad Nacional de Arqueología de Bolivia, en particular, a Javier Escalante y Pablo Rendón. A Gilles Rivière, Anita Cook e Ina Rössing por sus observaciones al texto. Finalmente, expreso mi profunda gratitud a los kallawaya y k'awayu por mostrarme el manejo de muchos objetos curativos descritos en el presente texto, especialmente a los kallawaya Walter Álvarez Quispe y Max Chura.

\section{Referencias citadas}

\section{Fuentes de archivo}

AUNAR (Archivo de la Unidad Nacional de Arqueología, La Paz)

1998a - Acta de recepción del material arqueológico, 1 f.; La Paz. 28 de enero.

1998b - Acta de recepción y apertura del material arqueológico, 2 fs.; La Paz. 2 de febrero.

AUNAR, Dirección Nacional de Antropología y Arqueología, Centro de Investigaciones Arqueológicas de Tiwanaku, Departamento de Conservación de la Dirección Nacional de Antropología y Arqueología, 1998c - Proyecto arqueológico de Amaguaya, 10 p.; La Paz. Abril de 1998.

1998d - Boletín informativo 030/98, 4 fs.; La Paz.

1998e - De Margaret Young-Sánchez, Associate Curator Art of the Americas, Africa and Oceania, al Lic. Jorge Velarde Chávez, Director General de Patrimonio Cultural del Viceministerio de Cultura de Bolivia, 2 fs.; La Paz. 30 de julio.

$1998 f$ - De Eduardo Pareja S., Jefe del Departamento de Conservación, al Lic. José Teijeiro V., Director Nacional de la Dirección Nacional de Arqueología, 2 fs.; La Paz. 16 de noviembre.

1999a - Fichas del registro del material arqueológico de Amaguaya (La Paz), 54 p.

1999b - «De José M. Capriles Flores al Lic. José Teijeiro Villarroel acerca de la identificación de elementos en especimenes del Proyecto Amaguaya» (La Paz, 1 de marzo de 1999), 4 fs.

1999c - «De Constantino Manuel Torres al Dr. Javier Escalante Moscoso, Director Nacional de Antropología y Arqueología» (Florida), $1 \mathrm{f}$. 
APGL (Archivo Privado Gregorio Loza; La Paz)

APGLa - De los arboles; frutos; aves y otras cosas medicinales... tiene este Reyno: siglo XVIII/ por Gregorio de Losa Avila y Palomares, 36 fs.; La Paz. Manuscrito.

APGLb - De la naturaleza, calidades y grados de arboles, flores animales y otras cosas exquisitas y raras del Nuevo Orbe del Perú y más claridad por orden A.B.C., 107 fs.; La Paz. Manuscrito.

APCBL (Archivo Privado Carmen Beatriz Loza)

Entrevista 4 - Max Chura, Kallawaya del ayllu Chari (La Paz, 5.XII.2005; El Alto 19.I.2007). Entrevista 5 - Walter Alvarez Quispe, Kallawaya del ayllu Canlaya (La Paz, 22.VI/30.XI.2005; Potosí, 23.III.2006). Entrevista 6 - Gerardo Alvarez Huanca, Kallawaya del ayllu Canlaya (Canlaya, 30.XI.2005). Entrevista 7 - Hugo Ticona, Kallawaya del ayllu Canlaya (Potosí, 2.III/23.III.2006). Entrevista 8 - Ramón Alvarez, Kallawaya del ayllu Canlaya (Chajaya, 20.XII.2005). Entrevista 9 - Paulina Chucamani, K'awayu del ayllu Sullkayana, Ukupampa (El Alto, 26.XI/6.XII.2005). Entrevista 10 - Víctor Saravia, K'awayu del ayllu Sullkayana (La Paz, 3.IV.2005 y K'awayu 1.I.2006). Entrevista 11 - Jesús Gómez, Kallawaya del ayllu Curva (Cochabamba, 20.XII.2005). Entrevista 12-Walter Quispe, Kallawaya del ayllu Curva (Cochabamba, 30.XII.2005). Entrevista 13 - Germán Montalvo Acarapi, K'awayu del ayllu Sullkayana, Ukupampa, (K'awayu, 29,3. XII. 2005 y Potosí, 20.III.2006). Entrevista 14 - Eusebia Mamani Saravia, K'awayu (Potosí, 29.XII.2005). Entrevista 15 - Fernando Illatarco, Kallawaya del ayllu Inca Roca (La Paz, 5.I.2006). Entrevista 16 - Ponciano Kahua, Kallawaya del ayllu Huata Huata (La Paz, 6.I.2006). Entrevista 17 - Cristina Saravia, K'awayu del ayllu Sullkayana (El Alto, 8.I.2006). Entrevista 18 - Nicolasa Saravia Quispe, K'awayu del ayllu Sullkayana (Potosí, 20.II.2006). Entrevista 19 - José LLanque, experto textil y mallku del ayllu Collana de K'ulta (Potosí, 3.II.2006). Entrevista 20 - David Keremba, Guaraní-Simba, capitanía Ingre, tenta Kasapa (Potosí, 20.IV/21.V.2006). Entrevista 21 - Florencio Hanco, K'awayu del ayllu Sullkayana, Paq'u de San Salvador (Potosí, 30.V.2006).

\section{Fuentes secundarias}

AGUILÓ, F., 1991 - Diccionario Kallawaya, 63 p.; La Paz: Museo Nacional de Etnografía y Folklore.

BERTONIO, L. J., 1989 [1612] - Vocabulario de la Lengua Aymara, 946 p.; La Paz: CERES, IFEA, MUSEF. Documentos históricos $N^{\circ} 1$, serie fuentes primarias $N^{\circ} 2$. Reimpresión facsimilar.

BUBBA, C., 1997 - Los rituales y los vestidos de María Titiqhawa, Juan Palla y otros fundadores de los "Ayllu" de Coroma. In: Saberes y memorias en los Andes. In memoriam Thierry Saignes (Thérèse Bouysse-Cassagne, ed.): 377-400; Paris: IFEA, Institut des Hautes Études de l'Amérique Latine de Paris. Coloquio que tuvo lugar en el Institut des Hautes Études de l'Amérique Latine de Paris III-Sorbona del 14 al 17 de febrero de 1994.

CAPRILES, J. M., 2002 - Intercambio y uso ritual de fauna por Tiwanaku. Análisis de pelos y fibras de los conjuntos arqueológicos de Amaguaya, Bolivia. Estudios Atacameños, 23: 33-50; San Pedro de Atacama. 
CAPRILES, J. M. \& FLORES, E., 2000 - Identificación de pelos y fibras animales procedentes del hallazgo arqueológico de Amaguaya. In: Anales de la XII Reunión Anual de Etnología, 1: 111-120; La Paz: Museo Nacional de Etnografía y Folklore.

CAPRILES, J. M. \& FLORES, E., 2002 - Identificación de pelos y fibras animales procedentes del hallazgo arqueológico de Amaguaya. Textos antropológicos, 11: 133-146; La Paz.

CHAPELLE, G., 1998 - Quels modèles pour la pensée? Poupées russes ou filet de pêche. Sciences Humaines, 89: 12-17; París.

CLARCK, N. R., PALACIOS, P. F. \& JUÁREZ, N., 1999 - Fardos textiles del Cementerio de Chiribaya baja, valle de llo (Andes centro-sur). Gaceta arqueológica andina, 25: 109-145; Lima.

CONDE, E., 1998 - Gran descubrimiento arqueológico investiga la DINAR. Cerámicas de 3400 años de antigüedad. Última Hora; La Paz. Sábado 1 de agosto.

DAVID, N. \& KRAMER, C., 2001 - Ethnoarchaeology in action, 476 p.; United Kingdom: Cambridge University Press.

DAVILA DE CANGAS, D. \& OTAZU, B. de, 1991 [1568-1569/1570] - Visita a los valles de Sonqo en los yunka de coca de La Paz (1568-1570), 687 p.; Madrid: ICl, Instituto de Cooperación Iberoamericana, Instituto de Estudios Fiscales. Edición a cargo de John Víctor Murra. Versión paleográfica de Rossana Barragán, Carmen Beatriz Loza y Felicity Nock.

EL DIARIO, 1998a - En la Cordillera Real de La Paz. Un importante hallazgo en el 98: Los restos precolombinos en Amaguaya. El Diario, año XCV, n 34680: 2; La Paz.

EL DIARIO, 1998b - Durante más de mil años. Piezas de Amaguaya se conservaron porque estuvieron aisladas del medio ambiente. El Diario, año XCV, n 34680: 2; La Paz.

FLORES OCHOA, J. A., 1977 - Pastores de puna: uywamichiq punarunaquna, 305 p.; Lima: Instituto de Estudios Peruanos.

GIRAULT, L., 1984 - Kallawaya: guérisseurs itinérants des Andes; recherches sur les pratiques médicinales et magiques, 668 p.; París: Institut Français de Recherche Scientifique pour le Développement en Coopération. Mémoires de l'Orstom 107.

GIRAULT, L., 1987 - Kallawaya: curanderos itinerantes de los Andes; investigación sobre prácticas medicinales y mágicas, 670 p.; La Paz: Institut Français de Recherche Scientifique pour le Développement en Coopération. Traducción al español de Carmen Bustillos y René Alcócer.

GUTIÉRREZ USILLOS, A., 1989 - Dioses, símbolos y alimentación en Los Andes. Interrelación hombre-fauna en el Ecuador prehispánico, 437 p.; Quito: Abya Yala.

HULTKRANTZ, ̊.., 2003 - The relation between medical status and soul beliefs. In: Medicine Across Cultures. History and Practice and Medicine in Non-Western Cultures (Helaine Selin, ed.): 385-395; Dordrechet/Boston/London: Kluwer Academic Publishers, Sciences Across Cultures.

INSTITUTO NACIONAL DE ESTADÍSTICA \& PNUD (Bolivia), 2005 - Atlas estadístico de Municipios 2005, 697 p.; La Paz: INE, PNUD.

KOSOK, P., 1965 - Life, land and water in ancient Peru. An account of the discovery, exploration and mapping of ancient pyramids, canals, roads towns, walls and fortresses of coastal Peru with observations of various aspects of Peruvian life both ancient and modern; New Cork: Long Island University Press.

LINDHOLM, C., 2000 - Chamán, chamanismo. In: Diccionario de antropología: 108; México: Siglo XXI. 
LOSA AVILA PALOMARES, G. de., 1983 [1780] - De los arboles; frutos; aves y otras cosas medicinales... tiene este Reyno: siglo XVIII/ por Gregorio de Losa Avila y Palomares; 285 p.; La Paz: Sociedad Geográfica de La Paz. Versión paleográfica, estudios y análisis por Gregorio Loza-Balsa.

LOZA-BALSA, G., 1995 - Esbozo de medicina aymara, 250 p.; La Paz: Gregorio Loza-Balsa.

LOZA, C. B., 2004 - Kallawaya Reconocimiento mundial a una ciencia de Los Andes, 250 p.; La Paz: Viceministerio de Cultura, UNESCO, Fundación del Banco Central de Bolivia.

LLAGOSTERA, A., 1988 - El complejo psicotrópico en SOLCOR-3 (San Pedro de Atacama). Estudios Atacameños, 9: 61-98; San Pedro de Atacama.

LLAGOSTERA, A., 2006 - Contextualización e íconografía de las tabletas psicotrópicas Tiwanaku de San Pedro de Atacama. Chungara, 38(1): 83-111; Arica.

OBLITAS POBLETE, E., 1955 - El machaj-juyai o idioma Callawaya. Khana, IV(9-10): 122129; La Paz.

OBLITAS POBLETE, E., 1956 - iSe ha descubierto el idioma sagrado de los Incas? Khana IV(17-18): 249-254; La Paz.

OBLITAS POBLETE, E., 1978 - Cultura Callawaya, 556 p.; La Paz: Ed. Populares Camarlinghi.

PAULSEN, A. C., 1974 - The Thorny Oyster and the Voice of God: Spondylus and Strombus in Andean Prehistory. American Antiquity, 39(4): 597-607; Washington D.C.: Society for American Archaeology.

POSNANSKY, A., 1945 - The craddle of American man, 2 vol.; New York: Augustin.

PRESENCIA, 1998a - En Amaguaya. Hallazgo abre puertas de Tiwanaku. Presencia: 3; La Paz. Martes 11 de agosto.

PRESENCIA, 1998b - Arqueología tiwanakota. Hallazgo de Amaguaya tiene casi mil años de antigüedad. Presencia: 3; La Paz. Martes 11 de agosto.

RENDÓN, P., 2000 - La tableta de rapé de Amaguaya. In: Anales de la XII Reunión Anual de Etnología, 1: 89-95; La Paz: Museo Nacional de Etnografía y Folklore.

RIVERA CUSICANQUI, S., 1986 - Oprimidos pero no vencidos: luchas del campesinado aymara y quechua 1900-1980, 201 p.; La Paz: Ed. Hisbol.

RÖSSING, I., 1990 - Introducción al mundo Kallawaya. Curación ritual para vencer penas y tristezas, 324 p.; Cochabamba: Editorial Los Amigos del Libro.

RÖSSING, I., 1992 - La mesa blanca Callawaya. Introducción, 292 p.; Cochabamba: Editorial Los Amigos del Libro.

RÖSSING, I., 1995 - La mesa blanca Callawaya, contribución al análisis, observaciones intraculturales y transculturales, 330 p.; Cochabamba: Editorial Los Amigos del Libro.

STARK, L., 1972 - Machaj-juyai: secret language of the Callahuayas. In: Papers in Andean Linguistics, 1(2):199-227; Madison/Wisconsin.

TORERO, A., 1987 - Lenguas y pueblos altiplánicos en torno al siglo XVI. Revista Andina, 5 (10): 329-405; Cusco.

TORRES, C. M., 1986 - Tabletas para alucinógenos en Sudamérica: Tipología, distribución y rutas de difusión. Boletín del Museo Chileno de Arte Precolombino, 1: 37-53; Santiago de Chile.

TORRES, C. M., 1996 - Archaeological evidence for the antiquity of psychoactive plant use in the central Andes. Annali del Museo Civico di Storia Naturale, 11: 291-326; Génova.

TORRES, C. M., 2001 - Iconografía Tiwanaku en la paraphernalia inhalatoria de los Andes Centro-Sur. Boletín de Arqueología de la Pontificia Universidad Católica del Perú, 5: 427-454; Lima. 
WASSÉN, S. H., 1972 - A medicine-man's implements and plants a Tihuanacoid Tomb in Highland Bolivia, 196 p.; Göteborg: Göteborgs Etnografiska Museum.

WERNER, M. \& ZIMMERMANN, B., 2004 - Penser I'histoire croisée : entre empirie et réfléxivité. In : De la comparaison à l'histoire croisée (Michel Werner \& Bénédicte Zimmermann, eds.) : 15-49; París : Seuil.

ZORN, E., 1987 - Un análisis de los tejidos en los atados rituales de los pastores. Revista Andina, 5: 489-526; Cusco. 